Int. J. Odontostomat.,

4(2):185-188, 2010.

\title{
Recursos Web para el Mejor uso de las Suscripciones BEIC - Chile
}

\author{
Web Resources for the Best use of BEIC - Chile Subscriptions
}

Cartes-Velásquez, Ricardo

\begin{abstract}
CARTES-VELÁSQUEZ, R. Recursos web para el mejor uso de las suscripciones BEIC-Chile. Int. J. Odontostomat., 4(2):185-188, 2010.

RESUMEN: Con la aparición de las prácticas basadas en la evidencia, se hace cada día más necesario permanecer actualizado con los avances que tiene nuestra profesión en sus diversas áreas. Lo anterior supone poder acceder a las fuentes primarias de esta información, como son los journals, los que muchas veces al ser de pago implican una gran barrera para su acceso, afortunadamente gracias una iniciativa de CONICYT y CINCEL las universidades chilenas cuentan con acceso a la mayoría de los principales journals en todas las áreas del conocimientos a través de la Biblioteca Electrónica de Información Científica (BEIC-Chile). El objetivo de este artículo es mostrar una forma sencilla, gratuita y basada en la web para el manejo de las suscripciones que podemos tener a los journals de nuestro interés, exponiendo el uso de la plataforma Google Reader y las extensiones Google Reader Watcher y FoxyProxy del navegador Mozilla Firefox, tanto para su uso específico en BEIC-Chile, como así otras posibles aplicaciones de estos recursos al interior de las instalaciones universitarias y fuera de ellas. Es necesario conocer y aplicar las diversas herramientas tecnológicas con las que contamos en la actualidad, así como también aprovechar plataformas como BEIC-Chile, las que nos permiten entregar prestaciones de mejor calidad a nuestros pacientes.
\end{abstract}

PALABRAS CLAVE: odontología basada en la evidencia, BEIC, recursos web, Mozilla Firefox, CINCEL.

\section{INTRODUCCIÓN}

El advenimiento de la medicina basada en la evidencia (MBE) hace casi 2 décadas (Letelier \& Moore, 2003) exige que todos los profesionales de la salud se mantengan actualizados constantemente en los últimos avances de sus distintas especialidades, en este sentido la información proveniente de las revistas científicas arbitradas por pares sigue siendo considerada la fuente principal y más confiable de dicha información junto, por supuesto, a bases con una orientación específica al MBE como la Colaboración Cochrane. Sin embargo, lo que si ha variado es la forma en que estas publicaciones llegan a los lectores, que con la aparición de la web han comenzado una transición desde el papel a las pantallas de computadora, cambio que podríamos considerar congénito en muchas revistas que aparecen única y exclusivamente en su edición electrónica, ejemplos notables en este sentido son las que se agrupan bajo BMC, PLoS y otras similares.
Pero la publicación electrónica no solo significa un cambio en el soporte, ha significado también una enorme modificación en el acceso, audiencias, relaciones económicas, impactos, reputación académica y un sinfín de aspectos que forman parte de la divulgación científica; es en este cambio que han nacido o se han potenciado términos como "open access", "publish-or-perish", "factor de impacto" y hasta la misma MBE entre otros (Bosch, 2009).

En relación a las audiencias o los consumidores de ciencia, comunidades académicas y/o de investigación, crecientemente acceden de manera electrónica y la mayoría de las principales revistas científicas cuentan con una edición electrónica (distinto ISSN) o digitalizan su versión en papel, las que mayoritariamente son albergadas por editoriales transnacionales como Elsevier, Wiley, Springer y otras, las que además de ofrecer los contenidos propiamen- 
te tal, ponen a disposición servicios anexos de búsqueda, artículos relacionados, canales RSS, alertas por mail, entre otros.

Aquí es donde se hace muy importante considerar como los centros universitarios otorgan el acceso a las revistas científicas y se ajustan a las nuevas condiciones, así como los usuarios (estudiantes, académicos e investigadores) maximizan los beneficios que este nuevo escenario les entrega. El objetivo de este artículo es mostrar un caso particular de lo previamente expresado: como de manera simple, efectiva y haciendo uso de herramientas web gratuitas podemos mantenernos actualizados gracias al acceso a la Biblioteca Electrónica de Información Científica (BEIC-Chile).

\section{BEIC-Chile}

El año 2002 la Comisión Nacional de Investigación Científica y Tecnológica (CONICYT) de Chile en conjunto con el Consejo de Rectores, que alberga a las 25 Universidades tradicionales del país, crearon el Consorcio para el Acceso a la Información Científica Electrónica (CINCEL) con el objetivo de dar respuesta a la creciente y dificultosa necesidad de mejorar el acceso a recursos de información científica que tenían las universidades chilenas (CINCEL, 2009). La corporación CINCEL desarrolló entonces una iniciativa conjunta y cofinanciada con CONICYT para la formación de BEIC, biblioteca que agrupa más de cinco mil revistas a texto completo, en más de cien áreas científicas, pertenecientes a ocho editoriales: Elsevier, Blackwell, Nature Publishing Group, Oxford University Press, Springer, American Chemical Society, Annual Reviews y Wiley. El acceso a estos títulos se hace a través de las redes IP de las universidades y centros de investigación participantes, pero además algunas permiten el acceso desde redes externas mediante servidores proxy, lo que facilita enormemente el trabajo de los investigadores.

\section{Uso de recursos web para el manejo de suscripciones en BEIC}

Es fácil recordar lo común que era, aún sigue siendo en muchos casos, como los clínicos se suscribían a las revistas de su especialidad, las que periódicamente llegaban por correo físico y les permitían mantenerse actualizados. Si bien en la actualidad es muy fácil mediante buscadores (como PubMed o los que integran ScienceDirect, Springer y otras plataformas) encontrar artículos para temas o casos específi- cos que se enfrentan en la práctica diaria, la suscripción sigue siendo un buen hábito que nos permite estar al tanto de una gran cantidad de información y profundizar en aquella que es de mayor interés o necesidad para el desempeño de nuestra especialidad.

Tal como dijimos previamente el escenario del acceso a las publicaciones ha cambiado y la suscripción no ha escapado a ello, en la actualidad existen una serie de herramientas que facilitan el proceso y por tanto es menester conocerlas, aplicarlas y disfrutar de sus beneficios. El caso que se expone consiste en la experiencia y uso diario que el autor tiene para manejar la suscripción a una serie de revistas, entre las que se incluyen varios títulos de pago presentes en BEIC, haciendo uso de recursos web gratuitos y fácil configuración. Para lo anterior se deben considerar 2 partes: el sistema de alerta y la identificación para el acceso.

\section{El sistema de alerta}

En la mayoría de los casos las revistas electrónicas ofrecen 2 posibilidades para avisar a sus audiencias respecto a la aparición de un nuevo número: e-mail y canal RSS. A pesar que ambos logran su objetivo, es este último quien permite manejar de manera más ordenada la suscripción, en este caso la recomendación es hacer uso de plataformas web como Google Reader que permite agrupar canales RSS (suscripciones) de un mismo tema o especialidad en carpetas.

Activar la suscripción es tan sencillo como clickear el link RSS de la revista o en el ícono respectivo que aparece en la barra de dirección del navegador si es que tenemos debidamente configurada nuestra cuenta Google Reader como lector RSS o podemos copiar la URL del RSS y agregarla directamente en la pagina del reader. Si estamos haciendo uso del navegador Mozilla Firefox, el que ofrece una inmensa variedad de herramientas para facilitar la investigación (Mena Diaz, 2009), podemos agregar además una extensión como GReader que nos avisa cuantos y de que suscripciones tenemos ítems, artículos en este caso, sin leer.

\section{La identificación para el acceso}

En el caso de BEIC el acceso a las publicaciones se hace mediante redes IP que son reconocidas por parte de las editoriales, permitiendo la visualización de los artículos a texto completo. El problema 
surge cuando estamos trabajando fuera de las redes universitarias reconocidas, por ejemplo desde nuestros hogares, esto se soluciona conectándose a un servidor proxy que "enmascara" nuestra IP permitiendo acceder de la misma forma como si navegáramos desde la universidad. Sin embargo, no es necesario ni deseable que todo el tráfico que generamos pase a través del proxy, aquí es cuando nuevamente con la ayuda de una extensión de Firefox podemos filtrar que solo el tráfico hacia las revistas de BEIC pasen por el proxy, una buena opción es Foxyproxy que permite la configuración de varios proxys, cada uno de ellos con reglas, las de utilidad en este caso son las listas blancas donde agregamos las direcciones web raíz de las plataformas como ScienceDirect o InterScience, con lo que podemos acceder al texto completo de todos los títulos que BEIC tiene en estas plataformas.

\section{Otros usos}

La utilización de los RSS no solo se limita a revistas que estén en BEIC o en plataformas de pago, otra buena opción es suscribirse a revistas open-acces, como por ejemplo las presentes en SciELO y que en su mayoría permiten la suscripción por RSS, hacer notar que muchas publicaciones además de ofrecer RSS para sus últimos números publicados han comenzado a ofrecer otros específicos para los artículos aceptados y que se encuentran en prensa, lo que aparece como una suscripción temprana a la normal.
En el caso del proxy no solo permitirá el acceso desde los RSS sino que también cuando, por ejemplo, estemos buscando un artículo en PubMed podamos acceder directamente al artículo a texto completo gracias a los links directos que este buscador ofrece hacia las distintas plataformas, siempre y cuando la publicación este incluida en BEIC.

Existen otras iniciativas similares a BEIC en otros países o centros universitarios (Duarte Barrionuevo, 2004) que permiten el acceso a artículos por vía electrónica y que pueden configurar las suscripciones a revistas de interés de manera similar al expuesto, no solo en el área de la salud sino en cualquier campo del conocimiento.

\section{Consideraciones finales}

Las posibilidades que nos brinda la web en conjunto a iniciativas como BEIC dan una respuesta de calidad, gratuita y oportuna a la necesidad de mantenerse actualizados, como así también motivan a los profesionales a desarrollar investigación. Sin embargo, estas posibilidades deben ser informadas y aprovechadas al máximo, de otra manera esfuerzos gubernamentales y/o institucionales pierden sentido y lo que es peor dejamos de cumplir con nuestra responsabilidad de otorgar prestaciones de salud de calidad a nuestros pacientes.

CARTES-VELÁSQUEZ, R. Web resources for the best use of BEIC-Chile subscriptions. Int. J. Odontostomat., 4(2):185188, 2010.

ABSTRACT: With the advent of evidence-based practices, it becomes increasingly necessary being updated with the progress that has our profession in its various areas. This means access to primary sources of this information, such as journals, which often suposse a big barrier to access due to payment policies, fortunately through an initiative of CONICYT and CINCEL, the chilean universities have access to most important journals in all areas of knowledge through the Electronic Library of Scientific Information (BEIC-Chile). The aim of this paper is to show a simple, free, web-based management for subscriptions to the journals we have in our interest, exposing the case of the platform Google Reader and the Mozilla Firefox extensions: Google Reader Watcher and FoxyProxy, for their specific use in BEIC-Chile, as well as other possible applications of these resources within the university facilities and outside them. It is necessary to know and implement the several technological tools that we have now, as well as platforms like BEIC-Chile, which allow us to deliver better quality services to our patients.

KEY WORDS: evidence-based dentistry, BEIC, web resources, Mozilla Firefox, CINCEL.

\section{REFERENCIAS BIBLIOGRÁFÍCAS}

Bosch, X. A reflection on open-access, citation counts, and the future of scientific publishing. Arch. Immunol. Ther. Exp., 57:91-3, 2009.
CINCEL. CONICYT, Chile. 2009. Disponible en: http:// www.cincel.cl/documentos/Corporacion/ memoria_2009.pdf (Consultado: 9 de junio del 2010). 
CARTES-VELÁSQUEZ, R. Recursos web para el mejor uso de las suscripciones BEIC-Chile. Int. J. Odontostomat., 4(2):185-188, 2010.

Duarte Barrionuevo, M. El Consorcio de Bibliotecas Universitarias Andaluzas. Boletín de la Asociación Andaluza de Bibliotecarios, 19(75-76):177-92, 2004.

Letelier, L. M. \& Moore, P. La medicina basada en evidencia. Visión después de una década. Rev. Méd. Chile., 131:939-46, 2003.

Mena Díaz, N. Firefox como herramienta para la gestión de la información. Acimed, 20(4):76-83, 2009.
Dirección para correspondencia:

Ricardo Cartes-Velásquez

Villa Ralco s/n, Alto Biobio.

CHILE

Telefono: $56-43-333822$

Email: cartesvelasquez@gmail.com

Recibido : 05-08-2010

Aceptado: 19-08-2010 